\title{
Diffusion and interactions of point defects in hard-sphere crystals
}

Berend van der Meer, Marjolein Dijkstra, and Laura Filion

Citation: The Journal of Chemical Physics 146, 244905 (2017);

View online: https://doi.org/10.1063/1.4990416

View Table of Contents: http://aip.scitation.org/toc/jcp/146/24

Published by the American Institute of Physics

\section{Articles you may be interested in}

Growth of defect-free colloidal hard-sphere crystals using colloidal epitaxy The Journal of Chemical Physics 146, 074903 (2017); 10.1063/1.4976307

Density functional theory and simulations of colloidal triangular prisms

The Journal of Chemical Physics 146, 124905 (2017); 10.1063/1.4978502

A simulation study on the phase behavior of hard rhombic platelets

The Journal of Chemical Physics 146, 144901 (2017); 10.1063/1.4979517

Thermodynamics of star polymer solutions: A coarse-grained study

The Journal of Chemical Physics 146, 244908 (2017); 10.1063/1.4989476

Periodic layers of a dodecagonal quasicrystal and a floating hexagonal crystal in sedimentation-diffusion equilibria of colloids

The Journal of Chemical Physics 147, 104902 (2017); 10.1063/1.4993521

Crystal nucleation as the ordering of multiple order parameters

The Journal of Chemical Physics 145, 211801 (2016); 10.1063/1.4962166

\section{AIP | The Jounal of Chemical Physics}




\title{
Diffusion and interactions of point defects in hard-sphere crystals
}

\author{
Berend van der Meer, Marjolein Dijkstra, and Laura Filion \\ Soft Condensed Matter, Debye Institute for Nanomaterials Science, Utrecht University, Princetonplein 5 , \\ 3584 CC Utrecht, The Netherlands
}

(Received 7 April 2017; accepted 14 June 2017; published online 29 June 2017)

\begin{abstract}
Using computer simulations, we study the diffusion, interactions, and strain fields of point defects in a face-centered-cubic crystal of hard spheres. We show that the vacancy diffusion decreases rapidly as the density is increased, while the interstitial diffusion exhibits a much weaker density-dependence. Additionally, we predict the free-energy barriers associated with vacancy hopping and find that the increasing height of the free-energy barrier is solely responsible for the slowing down of vacancy diffusion. Moreover, we find that the shape of the barriers is independent of the density. The interactions between vacancies are shown to be weakly attractive and short-ranged, while the interactions between interstitials are found to be strongly attractive and are felt over long distances. As such, we find that vacancies do not form vacancy clusters, while interstitials do form long-lived interstitial clusters. Considering the strain field of vacancies and interstitials, we argue that vacancies will hardly feel each other, as they do not substantially perturb the crystal, and as such exhibit weak interactions. Two interstitials, on the other hand, interact with each other over long distances and start to interact (attractively) when their strain fields start to overlap. Published by AIP Publishing. [http://dx.doi.org/10.1063/1.4990416]
\end{abstract}

\section{INTRODUCTION}

Defects are thermodynamically bound to occur in any crystal at finite temperature. These defects play an important role in the mechanical and transport properties of crystals and are a crucial factor for mechanical instabilities such as creep, yield, and fracture. ${ }^{1-4}$ The key aspects that underlie these phenomena are the concentrations at which these defects occur, their mobility, and their mutual interactions.

The hard-sphere model system is one of the most extensively investigated systems and has strongly contributed to a basic understanding of a variety of fundamental phenomena in condensed matter physics, such as glass transitions, ${ }^{5-7}$ crystal nucleation, ${ }^{8-13}$ and optimal packings. ${ }^{14-17}$ Likewise, hard spheres provide a simple model system to study crystal defects, both in statistical mechanical theories ${ }^{18-21}$ and in experiments using "colloidal" hard spheres. ${ }^{22-27}$ While these experiments on colloidal particles allow for direct quantitative studies of crystals in real space and real time, studying their defects is typically very challenging. Therefore, computer simulations have proven to be very useful. The first studies of point defects in hard spheres date back to the early 1970s by Bennett and Alder. ${ }^{28}$ Using computer simulations, they estimated a relatively high vacancy concentration close to melting $\left(\sim 10^{-4}\right)$. Moreover, they showed that the concentrations of higher-order vacancies, such as di-vacancies and tri-vacancies, are significantly smaller than those of mono-vacancies. Almost three decades later, Pronk and Frenkel calculated the equilibrium concentrations of both vacancies and interstitials, even for polydisperse systems. ${ }^{18,19}$ More recently, Lechner provided a method to calculate the effective interactions between point defects in two-dimensional crystals, which were shown to be attractive in a system of soft, dipolar spheres. ${ }^{29,30}$
Yet, so far a detailed understanding of the dynamics and mutual interactions of point defects in hard-sphere crystals is lacking.

In this paper, we study the diffusion, interactions, and strain fields of point defects in a face-centered-cubic hardsphere crystal. We show that the vacancy diffusion decreases rapidly as the density is increased, while the interstitial diffusion exhibits a much weaker density-dependence. The interactions between vacancies are shown to be weakly attractive and short-ranged. As such, di-vacancies are found to be unstable and break up easily into two separate mono-vacancies, which may occasionally reform into a di-vacancy. The interactions between interstitials, however, are found to be strongly attractive and are felt over long distances. As such, we find that interstitials form stable interstitial clusters, which only sporadically manage to dissociate into separate mono-interstitials. These point defect interactions can be understood by considering the strain field of vacancies and interstitials.

\section{MODEL AND METHODS}

\section{A. Simulation details}

We investigate face-centered-cubic crystals of $N_{L}$ lattice sites and $N$ hard spheres of diameter $\sigma$ using event-driven molecular dynamics (EDMD) simulations. We express the density in terms of the number of lattice sites per unit volume, i.e., $\rho=N_{L} / V$, where $V$ is the volume of the simulation box. Note that in these reduced units, the coexistence densities are $\rho_{s} \sigma^{3}=1.0372$ for the solid and $\rho_{f} \sigma^{3}=0.9387$ for the fluid. ${ }^{31}$ We define the EDMD unit time as $\tau=\sqrt{\beta m \sigma^{2}}$, where $m$ is the mass of a particle, and $\beta=1 / k_{B} T$ with $k_{B}$ Boltzmann's constant and $T$ the temperature. 
For the calculations of the free-energy barrier associated with vacancy diffusion and the calculations of the vacancyvacancy interactions, we have also employed Monte Carlo (MC) simulations. For the latter, the incorporation of "hopping" moves allows for more efficient sampling of all separation distances at high densities. More specifically, apart from regular translational moves of particles, we also allow a particle to move an integer number of lattice spacings, thus greatly enhancing the probability that a particle will jump into the vacancy.

\section{B. Defect tracking}

We locate vacancies and interstitials in the crystal using algorithms similar to those mentioned in Refs. 18 and 29. Namely, we assign each particle to its closest lattice site and check the occupancy of each lattice point. If there are no particles assigned to a given lattice point $\mathbf{R}$, it corresponds to a vacancy defect. We define the vacancy position to be equal to the position of the empty lattice site: $\mathbf{r}_{\mathrm{vac}}(t)=\mathbf{R}$. If there are two particles assigned to a given lattice point, it corresponds to an interstitial defect. In this case, we calculate for both particles the distance to the lattice point and define the interstitial position to be equal to the position $\mathbf{r}_{i}$ of the particle $i$ that is furthest away from the lattice point: $\mathbf{r}_{\text {int }}(t)=\mathbf{r}_{i}$. Note that during the simulation we correct for the center of mass drift of the system, as described in Ref. 32.

\section{Initialization of point defects}

In all simulations, we start from a lattice in which we have introduced the desired number of vacancies or interstitials. Vacancies are initialized by simply removing random particles from an otherwise perfect crystal. The introduction of interstitials, on the other hand, can be a bit more involved at high densities. To this end, we start from a low density crystal, in which the interstitials can be introduced without creating overlaps, and compress it to the desired density. This is accomplished using standard NPT MC simulations, in which the number of particles $N$, the pressure $P$, and the temperature $T$ are kept constant.

\section{RESULTS}

\section{A. Diffusion coefficients of vacancies and interstitials}

We begin by examining the diffusion of vacancies and interstitials. To this end, we introduce either one vacancy or one interstitial in the crystal and follow the diffusion of the point defect using the tracking algorithm described in Sec. II B. From these trajectories, we calculate the diffusion constant of the defect from the long-term diffusive behaviour of the mean square displacement,

$$
D=\lim _{t \rightarrow \infty} \frac{\left\langle\Delta r^{2}(t)\right\rangle}{6 t},
$$

where $\Delta r^{2}(t)=|\mathbf{r}(t)-\mathbf{r}(0)|^{2}$ with $\mathbf{r}(t)$ the position of the defect at time $t$. We plot the diffusion constants for vacancies and interstitials as a function of density in Fig. 1. For vacancies, we find that diffusion goes down rapidly with the density $\rho$, in agreement with the early results by Bennett and Alder. ${ }^{28}$ This is understandable as in order for the vacancy to diffuse, a

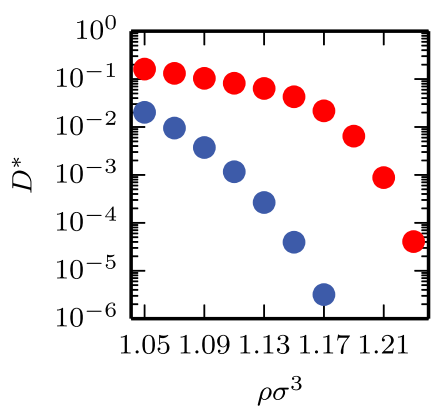

FIG. 1. Vacancy (blue) and interstitial (red) diffusion constants $D^{*}$ as a function of the density $\rho$. The diffusion constants were rendered dimensionless using $D^{*}=D \tau \sigma^{-2}$.

neighbouring particle has to hop into it, which becomes increasingly more difficult at higher densities. In contrast, for interstitials we observe a weak density-dependence on the mobility for densities $\rho \sigma^{3} \lesssim 1.17$ and only observe a more drastic slowing down at very high densities $\rho \sigma^{3} \gtrsim 1.19$. These data thus show that the slowing down of vacancies and interstitials is fundamentally different from each other and does not follow the same trend. We also observe that interstitial diffusion is always faster than vacancy diffusion, $D_{i}(\rho)>D_{v}(\rho)$. Thus, there is a density window where we expect vacancies to be essentially immobile while interstitials are still mobile.

\section{B. Free-energy barrier for vacancy diffusion}

To better understand the rapid slowing down of vacancy diffusion with increasing density $\rho$, we calculate the freeenergy barrier associated with vacancy hopping. More specifically, we introduce a single vacancy in the crystal and constrain all but one particle to their own lattice point. This one particular particle is allowed to hop between its own lattice site and the neighbouring vacant lattice site. By projecting the positions of this hopping particle onto the line that connects the two lattice sites, we obtain the free-energy barrier using $\beta F(x)=-\ln (P(x))$ with $P(x)$ the probability distribution function of the projected particle coordinate $x$. Here $x= \pm \frac{1}{2}$ corresponds to the particle being located at one of the lattice sites, and $x=0$ corresponds to the transition state. This is sketched in Fig. 2(a).

In Fig. 2(b), we show the predicted free-energy barriers associated with vacancy hopping for a range of densities. These free-energy profiles confirm that the height of the free-energy barrier increases strongly with increasing density. Interestingly, we observe no changes in the shape of the free-energy barriers. To show this, we normalize all free-energy profiles by their maximum barrier height $\beta F^{*}$, and obtain an excellent collapse, as shown in Fig. 2(c).

The increasing height of the free-energy barrier for vacancy hopping is solely responsible for the slowing down of vacancy diffusion. In Fig. 2(d), we plot the activation energy $\beta F^{*}$ versus the vacancy diffusion coefficient $D_{v}$, and obtain a clear exponential dependence.

\section{Interactions between point defects}

Next, we quantify the interactions between point defects. Our method is based on recent simulation studies of the 
(a)
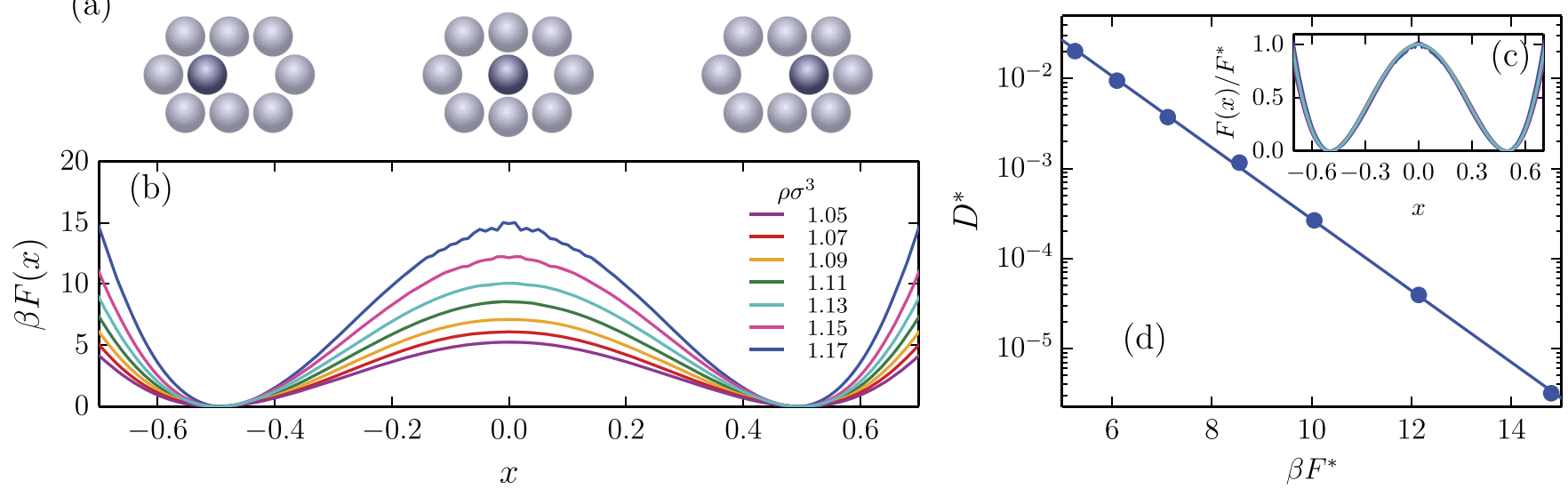

FIG. 2. Free-energy barriers associated with vacancy diffusion. (a) A schematic picture of a hopping vacancy showing a particle at the left lattice site $x=-\frac{1}{2}$, at the transition state $x=0$, and at the right lattice site $x=\frac{1}{2}$. (b) The free-energy barrier for vacancy diffusion $\beta F(x)$ for a range of densities. (c) The normalized free-energy barrier for vacancy diffusion, which collapses for many different densities to a single profile. (d) The exponential dependence of the vacancy diffusion constant $D^{*}$ on the barrier height $\beta F^{*}$.

interactions between point defects in two-dimensional colloidal systems of dipolar spheres. ${ }^{29,33}$ Here, we apply this method to hard-sphere crystals in three dimensions.

We start off by introducing either two vacancies or two interstitials into the crystal and follow their motion over time. In the case of vacancies, the separation distance between the two empty lattice sites located at positions $\mathbf{R}_{i}^{\mathrm{vac}}$ and $\mathbf{R}_{j}^{\mathrm{vac}}$ is given by $r=\left|\mathbf{R}_{i}^{\mathrm{vac}}-\mathbf{R}_{j}^{\mathrm{vac}}\right|$. The separation distance $r$ can now be used to define an effective potential as

$$
\beta F(r)=-\ln \frac{P(r)}{n_{L}(r)},
$$

where $P(r)$ is the probability to find the vacancy pair at a separation distance $r$ and $n_{L}(r)$ is the number of lattice sites at a distance $r$ from a reference lattice site. In the case of interstitials, we choose to express the separation distance also in terms of the distance between the two doubly occupied lattice sites $r=\left|\mathbf{R}_{i}^{\text {int }}-\mathbf{R}_{j}^{\text {int }}\right|$, where $\mathbf{R}_{i}^{\text {int }}$ and $\mathbf{R}_{j}^{\text {int }}$ refer to the positions of the two doubly occupied lattice sites.

The effective potential for the vacancy-vacancy interaction is shown in Fig. 3(a) for a range of densities $\rho$. Clearly the vacancy-vacancy interactions are only weakly attractive for all densities $\left(\approx-1 k_{B} T\right)$. These weak attractions highlight that indeed mono-vacancies do not form stable vacancy clusters, in agreement with the early results of Bennett and Alder, ${ }^{28}$
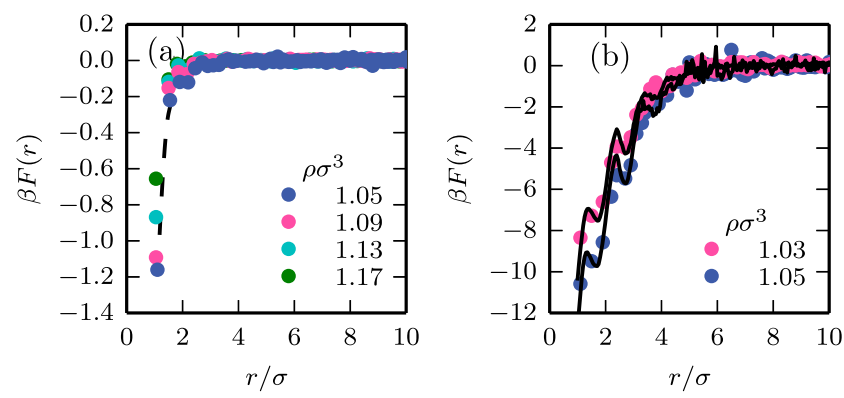

FIG. 3. Effective interactions between two vacancies (a) and two interstitials (b). The dashed black line in (a) is a guide to the eye. The solid black line in (b) corresponds to the interstitial-interstitial interaction potential as obtained from a $g(r)$-inversion. who showed that the concentrations of higher-order vacancies are significantly smaller than those of mono-vacancies. During the simulation, we observe that a di-vacancy will occasionally form from the fusion of two mono-vacancies, but these clusters break up continually. Thus entropy alone cannot stabilize vacancy clusters but rather tends to stabilize monovacancies due to the large number of possible configurations and its associated combinatorial entropy. Interestingly, the vacancy-vacancy interactions weaken slightly with increasing density; yet we observe very little density-dependence in the vacancy-vacancy interactions [Fig. 3(a)].

We also show the effective potential for the interstitialinterstitial interactions for varying densities $\rho$ [Fig. 3(b)]. In contrast to the vacancies, the interactions between interstitials are found to be strongly attractive and range many lattice sites. As such, we find that interstitials form long-lived di-interstitials, which only sporadically manage to dissociate into separate mono-interstitials. Thus entropy alone plays an important role in stabilizing interstitial clusters. We observe a large density-dependence on the interstitial-interstitial interactions. Namely, the effective interactions between interstitials become substantially stronger as the lattice becomes more compact with increasing density. Unfortunately, we were unable to properly sample the effective interactions at higher densities due to these strong interactions.

Interestingly, if we calculate $g(r)$ of the interstitial particle coordinates (instead of the interstitial lattice sites) and subsequently use $\beta F(r) \approx-\ln (g(r))$, we obtain an extremely similar effective potential (black solid line). This highlights that our way of calculating the effective interactions is robust and does not depend on the exact definition of the interstitial coordinate.

\section{Displacement field of point defects}

Intuitively, the interactions between defects arise from the strain that these point defects generate inside the crystal lattice. To this end, we calculate the average displacements around a single vacancy and a single interstitial, as summarized in Fig. 4. 


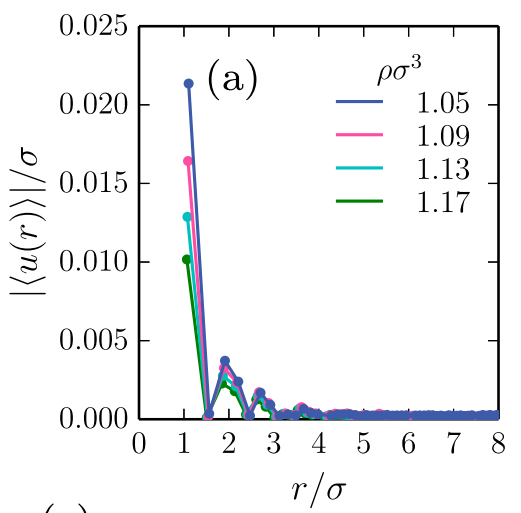

(c)
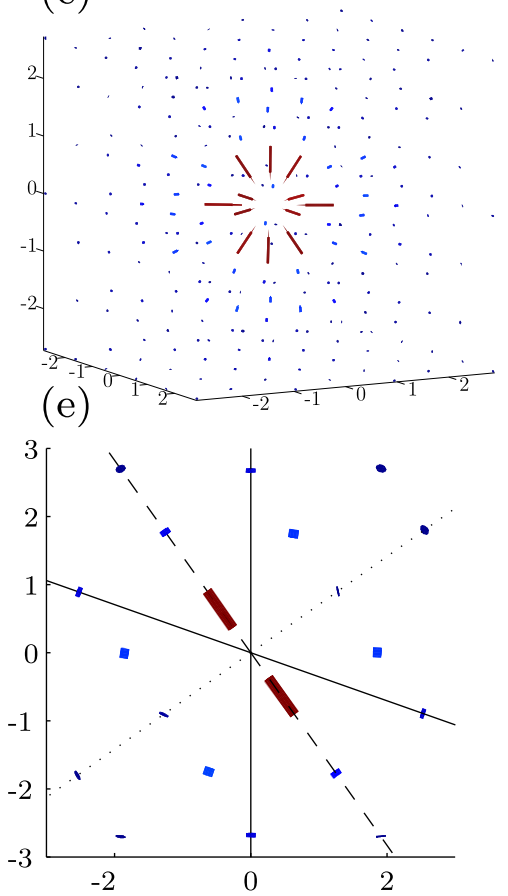

$----<110>$

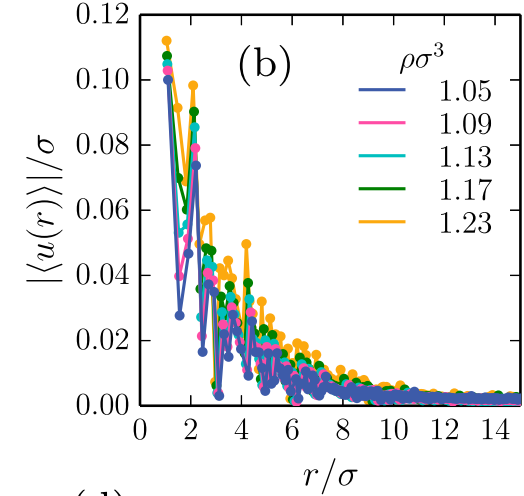

(d)

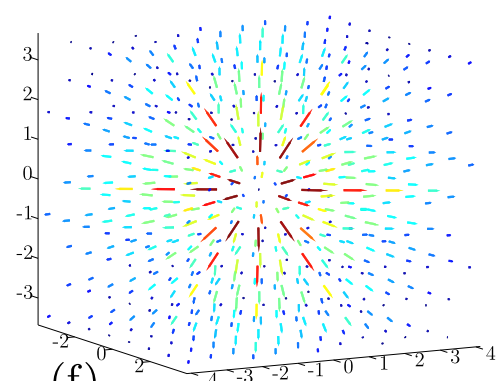

(f)

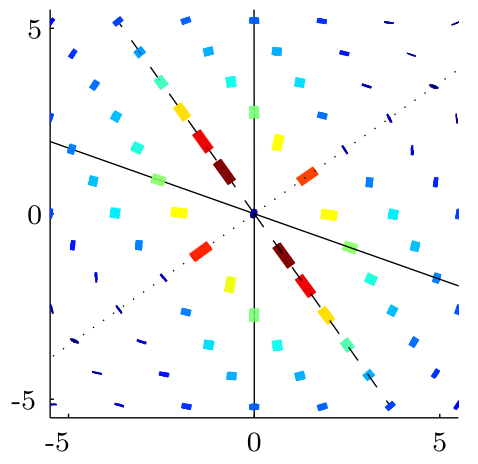

$<100>$
FIG. 4. The average displacements $|\langle u(r)\rangle|$ around a vacancy (a) and an interstitial (b) for various densities. (c) The displacement field $\langle\mathbf{u}(\mathbf{R})\rangle$ around a vacancy at $\rho \sigma^{3}=1.05$. (d) The displacement field $\langle\mathbf{u}(\mathbf{R})\rangle$ around an interstitial at $\rho \sigma^{3}=1.23$. (e) The displacement field $\langle\mathbf{u}(\mathbf{R})\rangle$ in the $\{110\}$-plane around a vacancy at $\rho \sigma^{3}=1.05$. (f) The displacement field $\langle\mathbf{u}(\mathbf{R})\rangle$ in the $\{110\}$-plane around an interstitial at $\rho \sigma^{3}=1.23$. In [(c) and (e)] and [(d) and (f)], displacement vectors are scaled up by a factor of 30 and 6 , respectively.
In the case of a vacancy, we observe that particles next to a vacancy tend to relax only a small amount $(0.01 \sigma-0.02 \sigma)$ inward towards the vacancy center, as shown in Fig. 4(a). Moreover, the displacements of particles are short-ranged; only the nearest neighbours feel the local dilation of the lattice. This is also clear upon plotting the average displacement vectors $\langle\mathbf{u}(\mathbf{R})\rangle$, as shown in Fig. 4(c), where vectors are scaled up by a factor of 30 . As such, we conclude that the vacancies hardly perturb the lattice. Note that with increasing density, the displacements around the vacancy decrease [Fig. 4(a)].

In contrast, interstitials cause substantial displacements of surrounding particles from their lattice sites: the nearestneighbouring particles are forced to displace large amounts from their lattice sites in order to be able to accommodate the interstitial particle. These particle displacements occur even over large distances from the core of the defect; the local deformation of the lattice is felt over many lattice sites from the interstitial center, as shown in Fig. 4(d). The displacements increase with increasing packing fraction [Fig. 4(b)]. Note that in the region near the center of the interstitial
( $r \lesssim 10 \sigma$ ), the displacements decay exponentially, as was also observed previously in a simple bead-spring model. ${ }^{34,35}$ Thus, somewhat surprisingly we find similar scaling to the beadspring model, despite the presence of hard interactions in our system.

From Figs. 4(c) and 4(d) it is clear that the displacements for both interstitials and vacancies are more pronounced along certain lattice directions than others. Thus, what appears as noise in Figs. 4(a) and 4(b) actually stems from anisotropic strains inside the lattice. This anisotropy is clearly visible in plots of the displacements in $2 \mathrm{~d}$ planes that pass through the defect. In Figs. 4(e) and 4(f) we plot such a plane, namely, the $\{110\}$-plane that intersects the defect. This plane allows us to examine the strain along the $\langle 110\rangle,\langle 100\rangle$, and $\langle 111\rangle$ directions, among others. Interestingly, these plots show that both for vacancies and interstitials, the displacements are most pronounced along the $\langle 110\rangle$-direction, while displacements along the other primary crystal axes, $\langle 100\rangle$ and $\langle 111\rangle$, are significantly weaker. Note that this anisotropic character to the strain fields is also responsible for the fluctuations in the interactions plotted in Fig. 3. 


\section{DISCUSSION AND CONCLUSIONS}

In conclusion, we have studied the diffusion, interactions, and strain fields of the simplest point defects in the hard-sphere model system. We have shown that the vacancy diffusion decreases rapidly as the density is increased, while the interstitial diffusion exhibits a much weaker density-dependence. The rapid decrease of the vacancy diffusion was found to be directly related to the increase in the height of the predicted one-dimensional free-energy barriers. Additionally, we have quantified the interactions between vacancies, which were shown to be weakly attractive and short-ranged, and between interstitials, which were found to be strongly attractive and act over much larger distances. Thus we found that entropy alone cannot stabilize vacancy clusters but rather tends to stabilize mono-vacancies, while interstitials tend to cluster into long-lived multi-interstitials. We also measured the average particle displacements around a single vacancy and a single interstitial. For vacancies, only the neighbouring particles were found to displace a small amount towards the vacancy center, while for interstitials the particles' displacements are large and even involve particles that are many lattice sites away from the defect center. This is well reflected in the effective interactions we have calculated, which were found to be weak and short-ranged for vacancies and strong and wide-ranged for interstitials.

The fact that vacancy diffusion goes down rapidly with increasing density explains why vacancies in the dense, lower regions of sediments will not be able to anneal out, as observed in both experiments and simulations. ${ }^{36,37}$ For interstitials, we found a weaker density-dependence on the diffusion constant. This feature may be especially relevant under out-ofequilibrium conditions where the crystal is perturbed (e.g., radiation damage or through the application of optical tweezers) and point defects are generated: while the interstitials may still be able to diffuse to the boundaries and anneal out, the vacancies will be stuck inside the bulk of the crystal. We hypothesize that the reason that vacancies slow down so drastically is that they are perfectly commensurate with the lattice and do not impose any lattice distortions. Namely, at high densities the jumping particle requires significant displacements of the surrounding particles in order to be able to pass through. Yet, these fluctuations become increasingly less probable at higher densities. For interstitials, however, the surrounding particles are always forced to deviate from their lattice site, in order to be able to accommodate the extra particle, and small collective displacements can lead to diffusion of the interstitial defect. It would be interesting to investigate further whether, similar to the $2 \mathrm{~d}$ case, ${ }^{38-41}$ distinct topological configurations of single point defects can be identified and connected to defect diffusion and rotation.

Lastly, we would like to point out that the predicted freeenergy barriers associated with vacancy diffusion all collapse onto a single curve when normalized by the barrier height. For further research, it would be interesting to investigate where this collapse originates from.

\section{ACKNOWLEDGMENTS}

We acknowledge funding from the Dutch Sector Plan Physics and Chemistry and a NWO-Veni grant (NWO-VENI Grant No. 680.47.432). We would like to thank Marijn van Huis, Frank Smallenburg, and Michiel Hermes for useful discussions and carefully reading the manuscript.

${ }^{1}$ G. I. Taylor, Proc. R. Soc. A 145, 362 (1934).

${ }^{2}$ M. Polanyi, Z. Phys. 89, 660 (1934).

${ }^{3}$ E. Orowan, Z. Phys. 89, 605 (1934)

${ }^{4}$ J. P. Hirth and J. Lothe, Theory of Dislocations (Wiley, New York, 1982).

${ }^{5}$ A. van Blaaderen and P. Wiltzius, Science 270, 1177 (1995).

${ }^{6}$ E. R. Weeks et al., Science 287, 627 (2000).

${ }^{7}$ W. K. Kegel and A. van Blaaderen, Science 287, 290 (2000).

${ }^{8}$ U. Gasser et al., Science 292, 258 (2001).

${ }^{9}$ S. Auer and D. Frenkel, Nature 409, 1020 (2001).

${ }^{10}$ S. Auer and D. Frenkel, Phys. Rev. Lett. 91, 015703 (2003).

${ }^{11}$ A. Cacciuto, S. Auer, and D. Frenkel, Nature 428, 404 (2004).

${ }^{12}$ V. de Villeneuve et al., J. Phys.: Condens. Matter 17, S3371 (2005).

${ }^{13}$ E. Allahyarov, K. Sandomirski, S. Egelhaaf, and H. Löwen, Nat. Commun. 6, 7110 (2015).

${ }^{14}$ L. Filion and M. Dijkstra, Phys. Rev. E 79, 046714 (2009).

${ }^{15}$ J. K. Kummerfeld, T. S. Hudson, and P. Harrowell, J. Phys. Chem. B 112, 10773 (2008).

${ }^{16}$ P. I. O'Toole and T. S. Hudson, J. Phys. Chem. C 115, 19037 (2011).

${ }^{17}$ A. B. Hopkins, Y. Jiao, F. H. Stillinger, and S. Torquato, Phys. Rev. Lett. 107, 125501 (2011).

${ }^{18}$ S. Pronk and D. Frenkel, J. Phys. Chem. B 105, 6722 (2001).

${ }^{19}$ S. Pronk and D. Frenkel, J. Chem. Phys. 120, 6764 (2004).

${ }^{20}$ S. K. Kwak, Y. Cahyana, and J. K. Singh, J. Chem. Phys. 128, 134514 (2008).

${ }^{21}$ M. Mortazavifar and M. Oettel, Europhys. Lett. 105, 56005 (2014).

${ }^{22}$ P. Schall, I. Cohen, D. A. Weitz, and F. Spaepen, Science 305, 1944 (2004).

${ }^{23}$ P. Schall, I. Cohen, D. A. Weitz, and F. Spaepen, Nature 440, 319 (2006).

${ }^{24}$ N. Y. C. Lin et al., Nat. Mater. 15, 1172 (2016).

${ }^{25}$ T. O. Skinner, D. G. Aarts, and R. P. Dullens, Phys. Rev. Lett. 105, 168301 (2010).

${ }^{26}$ E. Maire et al., Phys. Rev. E 94, 042604 (2016).

${ }^{27}$ F. A. Lavergne, S. Diana, D. G. A. L. Aarts, and R. P. A. Dullens, Langmuir 32, 12716 (2016).

${ }^{28}$ C. Bennett and B. Alder, J. Chem. Phys. 54, 4796 (1971).

${ }^{29}$ W. Lechner and C. Dellago, Soft Matter 5, 2752 (2009).

${ }^{30}$ W. Lechner et al., Phys. Rev. E 88, 060402 (2013).

${ }^{31}$ C. Vega and E. G. Noya, J. Chem. Phys. 127, 154113 (2007).

${ }^{32}$ M. Marechal, U. Zimmermann, and H. Löwen, J. Chem. Phys. 136, 144506 (2012).

${ }^{33}$ W. Lechner, F. Cinti, and G. Pupillo, Phys. Rev. A 92, 053625 (2015).

${ }^{34}$ W. Lechner, E. Schöll-Paschinger, and C. Dellago, J. Phys.: Condens. Matter 20, 404202 (2008)

${ }^{35}$ W. Lechner and C. Dellago, Soft Matter 5, 646 (2008).

${ }^{36}$ K. Jensen et al., Soft Matter 9, 320 (2013).

${ }^{37}$ T. Dasgupta, J. R. Edison, and M. Dijkstra, J. Chem. Phys. 146, 074903 (2017).

${ }^{38}$ A. Pertsinidis and X. Ling, Nature 413, 147 (2001).

${ }^{39}$ A. Pertsinidis and X. Ling, Phys. Rev. Lett. 87, 098303 (2001).

${ }^{40}$ A. Libal, C. Reichhardt, and C. O. Reichhardt, Phys. Rev. E 75, 011403 (2007).

${ }^{41}$ W. Lechner et al., Phys. Rev. E 91, 032304 (2015). 